\title{
Endovascular Treatment of Large Vessel Occlusion Strokes Due to Intracranial Atherosclerotic Disease
}

\author{
Jin Soo Lee, ${ }^{\mathrm{a}}$ Seong-Joon Lee, ${ }^{\mathrm{a}}$ Ji Man Hong, ${ }^{\mathrm{a}}$ Francisco José Arruda Mont Alverne, ${ }^{\mathrm{b}}$ \\ Fabricio Oliveira Lima, ${ }^{\mathrm{c}}$ Raul G. Nogueira ${ }^{\mathrm{d}}$ \\ ${ }^{a}$ Department of Neurology, Ajou University Hospital, Ajou University School of Medicine, Suwon, Korea \\ ${ }^{b}$ Interventional Neuroradiology Service, Hospital Geral de Fortaleza, Fortaleza, Brazil \\ 'Neurology Service, Hospital Geral de Fortaleza, Fortaleza, Brazil \\ ${ }^{d}$ Department of Neurology and Neurosurgery, University of Pittsburg Medical Center, UPMC Stroke Institute, Pittsburg, PA, USA
}

Mechanical thrombectomy (MT) has become the gold-standard for patients with acute large vessel occlusion strokes (LVOS). MT is highly effective in the treatment of embolic occlusions; however, underlying intracranial atherosclerotic disease (ICAD) represents a therapeutic challenge, often requiring pharmacological and/or mechanical rescue treatment. Glycoprotein IIb/IIla inhibitors have been suggested as the best initial approach, if reperfusion can be achieved after thrombectomy, with angioplasty and/or stenting being reserved for the more refractory cases. In this review, we focus on the therapeutic considerations surrounding the endovascular treatment of ICAD-related acute LVOS.

Keywords Cerebral infarction; Intracranial embolism and thrombosis; Intracranial arteriosclerosis; Endovascular procedures; Angioplasty; Stents
Correspondence: Raul G. Nogueira Department of Neurology and Neurosurgery, University of Pittsburg Medical Center, UPMC Stroke Institute, C-400 PUH, 200 Lothrop Street, Pittsburgh, PA 15213, USA Tel: +1-412-647-8080 Fax: +1-412-647-8445 E-mail: raul.g.nogueira@icloud.com https://orcid.org/0000-0003-4532-153X

Received: April 15, 2021

Revised: November 17, 2021

Accepted: December 8, 2021

\section{Introduction}

Endovascular treatment (EVT) based on mechanical thrombectomy (MT) has become the gold-standard for patients with acute large vessel occlusion strokes (LVOS) in the intracranial vasculature. ${ }^{1,2} \mathrm{MT}$ is particularly efficacious in the treatment of embolic occlusions. However, in situ atherothrombotic occlusions are often encountered and, in this setting, the underlying atheroma cannot be resolved by MT alone. ${ }^{3-8}$ This significantly complicates the treatment of acute LVOS related to intracranial atherosclerotic disease (ICAD) often resulting in longer procedure times and poorer outcomes. ${ }^{9,10}$

Several rescue treatments approaches have been studied for the treatment of the residual stenosis following MT. However, it is thought that intracranial stenting remains discouraged among them, in part due to the negative results of the previous trials for secondary stroke prevention for symptomatic intra- cranial stenosis. ${ }^{11,12}$ Although there have been no randomized trials of acute intracranial stenting for underlying ICAD in LVOS, rescue mechanical treatment with balloon angioplasty and/or stenting is often necessary. Many retrospective studies have suggested that balloon angioplasty and/or stenting is feasible in acute LVOS and results in favorable angiographic and clinical outcomes with an acceptable safety profile (Table 1). ${ }^{13-18}$ However, it is important acknowledge the limits on the data quality including the lack of standardization of the definition of ICAD-related LVOS and the broad range of the reported outcomes. $^{13,17}$

Underlying ICAD cannot be easily predicted in acute LVOS prior to the EVT, making it difficult to plan the treatment and pharmacologically prepare the patient in anticipation to the actual intervention. Herein, we review the therapeutic considerations surrounding the EVT of ICAD-related acute LVOS including the etiopathogenic aspects of acute cerebrovascular 


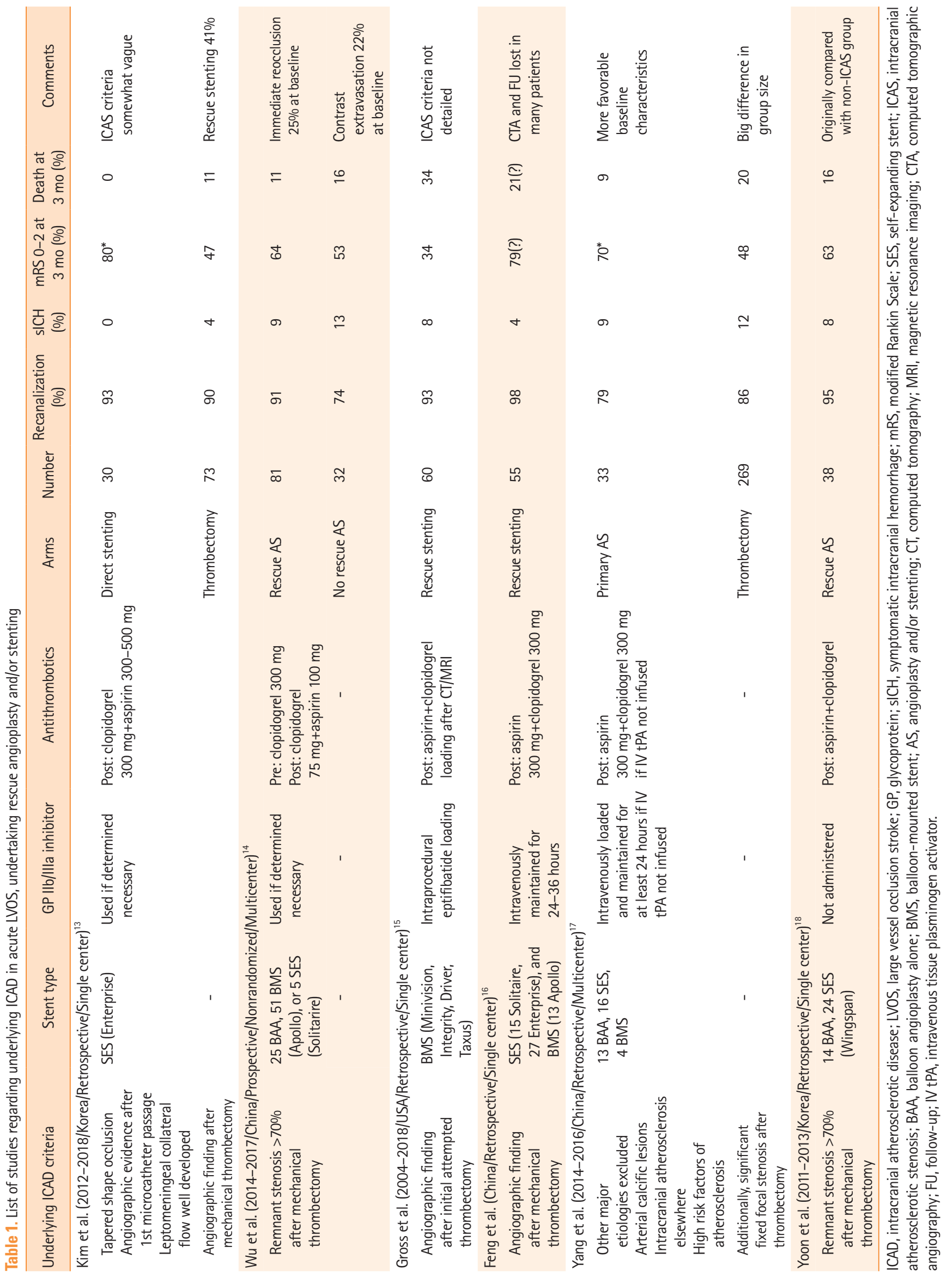


occlusions in general and the key pathophysiological and therapeutic considerations in ICAD-related acute LVOS.

\section{Etiopathogenic considerations in acute cerebrovascular occlusions}

There are many causes of cerebral infarction secondary to vascular occlusion of the brain (Figure 1). Representatively, it is divided into arteriosclerosis, cardioembolism, and small vessel disease. Currently, MT is indicated for selected patients presenting with acute LVOS up to 24 hours from stroke onset with an estimated incidence of approximately 10\% of all cerebral infarctions. A population-based study in the United States revealed large vessel occlusion (LVO) accounted for 324 patients (11.8\%) among 2,739 patients with acute ischemic stroke discharged from primary and secondary service areas, among whom 184 patients (6.7\%) presented with favorable Alberta Stroke Program Early CT Score (ASPECTS) $\geq 6$ in the anterior circulation. ${ }^{19}$

The etiology of LVO is simpler than the distribution of causes for overall cerebral infarctions. Cardiogenic or cryptogenic embolism is the most frequent cause, followed by large artery atherosclerosis (LAA) among acute LVOS. ${ }^{6}$ LAA can be further divided into intracranial and extracranial. ${ }^{20}$ Extracranial atherosclerosis (ECAS) can cause cerebral infarction by two types although these types can be mixed: hemodynamic compromise by severe stenosis and artery-to-artery embolism by in situ thrombosis and distal embolization. Atheroembolic events from ECAS can also lead to intracranial LVO (e.g., tandem occlusions). ${ }^{21}$ Likewise, in situ thrombosis in ICAD can also cause acute LVOS. ICAD-related LVOS tend to more commonly present as borderzone or scattered infarct types versus the wedgeshaped territorial infarct type that is more often associated with acute embolic LVOS. ${ }^{22}$

ICAD should be differentiated from the broader intracranial stenosis category. First, intracranial stenosis can be caused by many vascular diseases other than ICAD including vasculitis, reversible cerebral vasoconstrictive syndrome, vasospasm, arterial dissection and as well as Moyamoya disease and Moyamoya syndrome (Figure 1). Second, ICAD is a term that infers the underlying etiopathogenesis, while intracranial stenosis is simply an imaging finding (Figure 2). Thus far, underlying stenosis in acute LVOS has been mostly determined by angiographical findings, including fixed focal stenosis on digital subtraction angiography (DSA) both during and after finishing EVT. ${ }^{23}$ The term intracranial atherosclerotic stenosis (ICAS) has also been broadly used in this context. ${ }^{10,24,25}$ Although an embolus may sporadically get lodged in the intracranial stenosis, most ICAD-related LVOS are presumed to be caused by in situ thrombosis. As such,

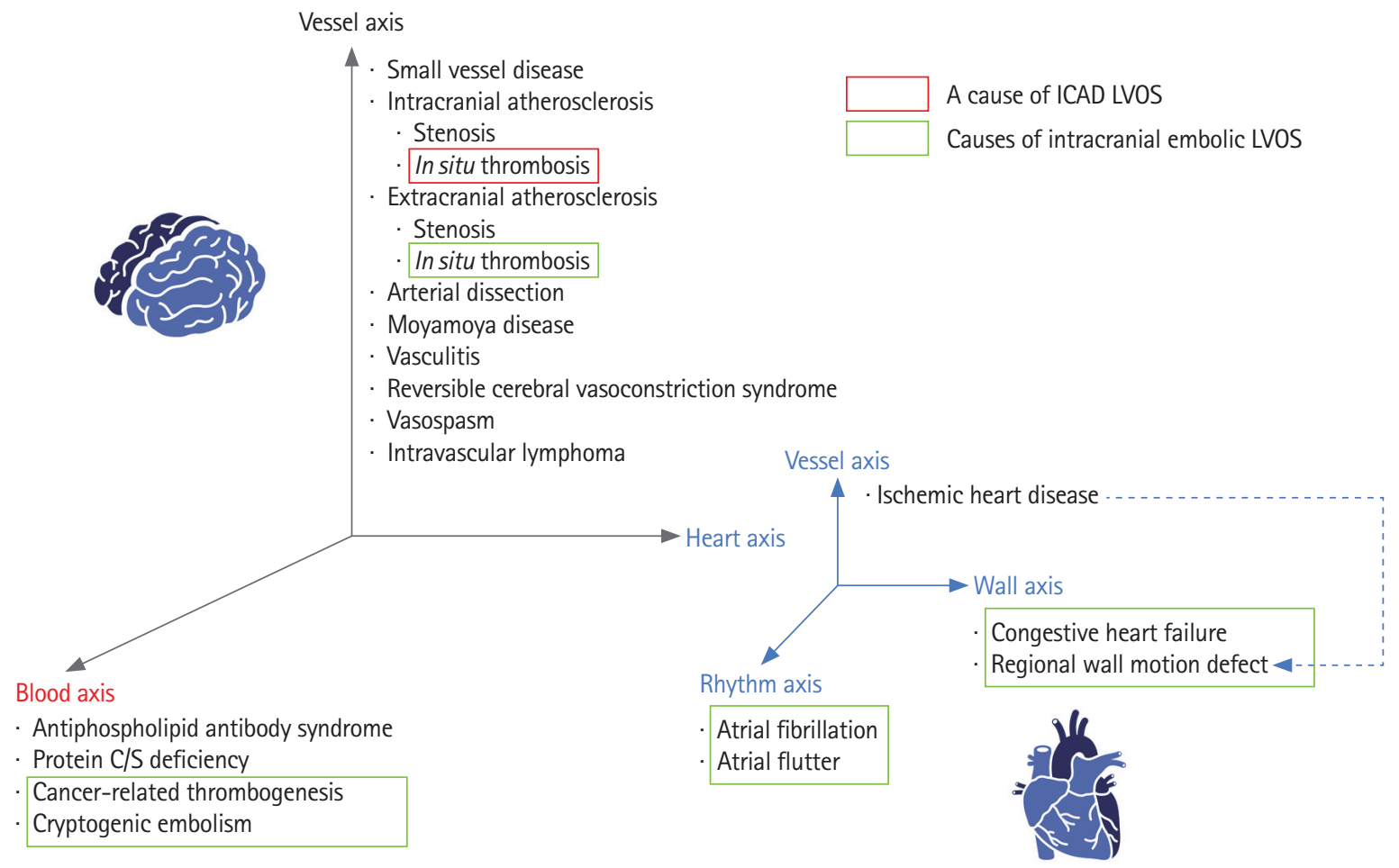

Figure 1. Etiologies of all cerebral infarctions and acute intracranial large vessel occlusion strokes (LVOS). The causes of cerebral infarction vary widely, while the causes of acute intracranial LVOS are relatively simple. Embolism from the heart, blood and extracranial atherosclerosis origins are the major causes of acute intracranial LVOS, followed by intracranial atherosclerotic disease (ICAD). 
Intracranial atherosclerotic stenosis as an angiographical term

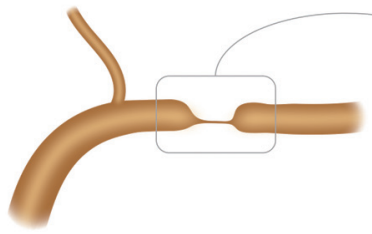

A severe stenosis

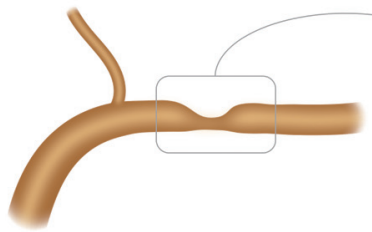

A moderate stenosis

\section{A}

Remaining stenosis after treatment

Intracranial atherosclerotic disease as a pathophysiological term
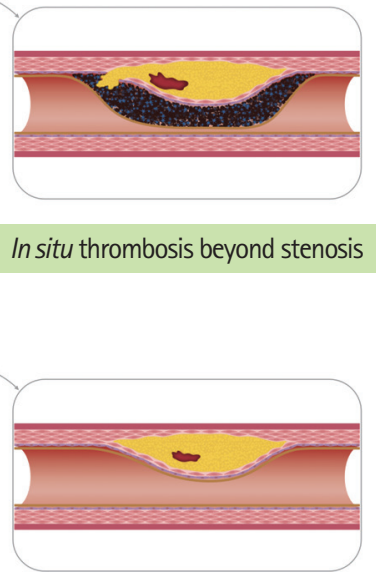

B

Figure 2. Schematic illustrations for subtle terminological differences between intracranial stenosis and intracranial atherosclerotic disease (ICAD). (A) Intracranial stenosis is an angiographical term. Although it can differentiate pathophysiology, its reading on digital subtraction imaging is a gold-standard for diagnosis. (B) From the aspects of pathophysiology, intracranial stenosis may consist of atherosclerosis itself and also in situ thrombi. Despite this aspect, pathological diagnosis is never practical. In this context, intracranial stenosis on angiography is generally referred as ICAD or intracranial atherosclerotic stenosis.

when it comes to the EVT of ICAD-related LVOS, one must consider the highly thrombogenic environment associated with an acutely inflamed atheromatous plaque. Third, several situations can be identified as vessel stenosis during revascularization treatment. Partially occlusive lesions can be seen if the culprit thrombus was partially resolved spontaneously or by the intravenous (IV) and/or endovascular interventions. In addition, stent retrieval can induce vasospasm, which may be confused with ICAS. The use of intra-arterial vasodilators such as nicardipine or milrinone may help differentiating the static ICAD lesion from the more dynamic vasospasm narrowing.

\section{Pathophysiological considerations in ICAD-related acute LVOS}

Despite the fact that ICAD-related acute LVOS and acute coronary syndrome share a common pathophysiology, namely, the rupture of atheromatous plaque with consequent in situ thrombotic occlusion, ${ }^{26,27}$ there are critical anatomical and technical differences when contemplating the EVT of these two entities. Acute stenting has been utilized for percutaneous coronary intervention (PCI) since the early 1980 s. $^{28}$ These devices

have been thoroughly developed along with adjunctive antiplatelet treatments, leading to the successful evolution in the implantation of self-expanding, balloon-expandable, and drug-eluting stents. ${ }^{29}$ Thus, along with coronary artery bypass graft surgery, $\mathrm{PCl}$ with stenting remains the mainstem in the care of coronary artery disease (CAD). ${ }^{30}$

There are several challenges to the direct translation of the $\mathrm{PCl}$ technology, pharmacology, and techniques into the intracranial territory. These are largely related to the distinct characteristics of the brain and heart at both the vascular and parenchymal levels. First, the intracranial arteries have unique morphological features. ${ }^{31}$ These arteries lack the external elastic lamina, which is normally located between the media and adventitia in other arteries. In addition, the media in intracranial arteries, which is composed of smooth muscle tissue and provides strength to the artery by allowing constriction and/or dilation, is thinner than in the other arteries in the body. From an interventional standpoint, this unique structure makes the intracranial vessels potentially more prone to complications, including vessel rupture, with angioplasty and/or stenting. In addition, intracranial vessels are further away from the arterial puncture site and have substantially more tortuosity than their coronary counterparts. This is particularly notorious at the level of the internal carotid artery siphon and terminus. This makes the navigation of balloon-mounted or higher radial force stents considerably more difficult. Finally, vascular recoil or restenosis may also be more challenging because of the smaller intracranial artery diameters.

Second, branch occlusion following angioplasty and/or stenting may cause serious neurologic deficits in the intracranial territory. Perforators in the middle cerebral and basilar arteries supply blood into the pyramidal tract and other highly eloquent neighboring brain structures. This means that angioplasty and/or stenting can recanalize the major artery trunk but may cause obstruction of perforators and lead to further neurological deficits, due to a phenomenon commonly referred as "snowplowing effect" where the expansion of the angioplasty balloon or stent mechanically displaces the atheroma into the ostia of side-branches. In the Stenting and Aggressive Medical Management for Preventing Recurrent Stroke in Intracranial Stenosis (SAMMPRIS) trial, in which percutaneous angioplasty and stenting with best medical treatment had poorer outcomes than best medical treatment alone ${ }_{1}{ }^{24}$ among the 19 patients with periprocedural ischemic complications, 12 patients had perforator occlusions, and another two patients had mixed perforator and embolic occlusions. ${ }^{32}$ In addition, the interventional treatment for the basilar artery, known to be a perforator-rich vessel, had a 6.2 odds ratio (OR) for periproce- 
dural ischemic events compared to other vessels. ${ }^{32}$ However, at least from a theoretical standpoint, angioplasty and stenting of an acute LVO may carry a lower risk of secondary ischemic injury from "snowplowing" in comparison to the "elective" treatment of chronic stenotic lesions since the vessel is not just narrowed but rather completely occluded and thus the territory supplied by the neighboring side-branches is often already infarcted as these are typically end-vessels deprived from any meaningful collateral supply.

Third, the brain parenchyma is a lot more prone to hemorrhagic transformation, ${ }^{33}$ complicating antithrombotic use, which is essential for preventing in stent thrombosis. In addition, antithrombotic use should be ideally avoided for 24 hours following intravenous fibrinolytic infusion, which remains the standard of care in patients with acute ischemic stroke presenting within 4.5 hours from stroke onset, with or without intracranial occlusion. However, even in cases with successful thrombolysis, immediate reocclusion might occur because the fibrinolytic agents may aggravate the prothrombotic tendency of the atherosclerotic lesion. ${ }^{34}$ This creates an important clinical dilemma as the use of antiplatelets in conjunction with intravenous thrombolysis (IVT) is off-label and might be associated with increased hemorrhagic risk. In the Antiplatelet therapy in combination with Recombinant t-PA Thrombolys in Ischemic Stroke (ARTIS) trial, intravenous administration of $300 \mathrm{mg}$ of aspirin within 90 minutes after intravenous alteplase, significantly increased the risk for early neurological deterioration due to the symptomatic intracranial hemorrhage $(\mathrm{sICH}) .{ }^{35}$ In the STARTING-SICH (systolic blood pressure, age, onset-to-treatment time for thrombolysis, NIHSS score, glucose, aspirin alone, aspirin plus clopidogrel, anticoagulant with INR $\leq 1.7$, current infarction sign, hyperdense artery sign [STARTING]-nomogram for predicting the probability of sICH) study, both the use of aspirin alone or in combination with clopidogrel were independent predictors of sICH in patients undergoing IVT. ${ }^{36}$ Notably, these data have been more recently contested by subgroup analyses of the Safe Implementation of Treatments in Stroke (SITS) and Thrombolysis in Stroke Patients (TRISP) registries, both demonstrating no added risks to IVT in patients who were taking dual antiplatelets (DAP). ${ }^{37,38}$

\section{Therapeutic considerations in ICAD- related acute LVOS}

We propose of multistep approach (SPRINT) for the treatment of ICAD-related acute LVOS as following: (1) Suspicion, (2) Pretreatment, (3) Retrieval, (4) Infusion of antiplatelets, (5) No-go decision, and (6) Treatment with angioplasty/stenting.

\section{Suspicion}

Unfortunately, there is no broadly accepted standardization for diagnosing underlying ICAD in acute LVOS before treatment. The Trial of Org 10172 in Acute Stroke Treatment (TOAST) classification, which is the most popular etiological classification system for secondary prevention, ${ }^{39}$ requires delaying the diagnosis to the post-treatment phase. Furthermore, some patients may require emergent angioplasty and/or stenting at time of MT so the follow-up imaging would be masked by the treatment. As such, we believe that an operation definition also including cases of acute reocclusion, vessel recoiling, or severe residual vascular narrowing refractory to intra-arterial vasodilators and requiring emergent angioplasty and/or stenting would be more suitable to clinical practice.

While baseline findings are often indistinguishable between embolic and atherothrombotic acute LVO, there are some demographic, clinical and imaging variables that may help differentiating across these two etiologies. Some factors such as age and ethnicity are risk factors for both atherosclerotic and cardioembolic events but, particularly in the absence of other known or suspected etiologies such as atrial fibrillation, dilated cardiomyopathy, or extracranial cervical stenosis, an increased suspicion for ICAD should occur in Asians, African-Americans, and Hispanics as well as diabetic patients. Nonetheless, it is important to acknowledge that cardioembolism, cryptogenic embolism, and ICAD are relatively common conditions and thus may coexist. ${ }^{6}$ Given the longer opportunity for collateral development, ICAD-related LVO may more often present with fluctuating or low-severity clinical symptoms as compared to corresponding embolic occlusions. For the same reason, these patients may be overrepresented in the late time window. ${ }^{10}$ Evaluation of the baseline parenchymal imaging on non-contrast computed tomography (CT) or magnetic resonance imaging may reveal acute, subacute, or chronic scattered or borderzone infarcts in the same territory of the acute LVO which are highly suggestive of ICAD versus larger territorial wedge-shaped infarcts which are more suggestive of embolic etiology, particularly when involving territories other than the occlusion site. ${ }^{22}$ The presence of calcification at the site of occlusion or the presence of severe intracranial carotid artery calcification favor underlying ICAD as the potential etiology whereas the presence of a dense vessels sign might favor an embolic lesion. ${ }^{40}$

The presence of severe and/or multifocal narrowing on CT angiography (CTA) strongly favors ICAD as the most likely etiology. Likewise, the occurrence of complete leptomeningeal collaterals on the baseline CTA is highly indicative of ICAD-related LVOS $(O R, 3.32 ; 95 \%$ confidence interval $[\mathrm{Cl}], 1.52$ to 7.26; $P=0.003$ ). ${ }^{41}$ The evaluation of the baseline CTA or of the 
DSA during first deployment of stent-retriever may disclose a (bifurcation sparing) truncal-type occlusion versus a (bifurcation involving) branching-site occlusion which favor ICAD and embolic etiologies, respectively. ${ }^{42-44}$ A Tmax profile of Tmax $>4$ seconds/Tmax $>6$ seconds ratio $\geq 2$ on CT perfusion reflects good collaterals and is also suggestive of ICAD-related LVO. ${ }^{45}$ As compared to "non-tapered" occlusions (such as those demonstrating a meniscus, a flat-edged cutoff, or a tram-track sign), "tapered" occlusions with a jet-like, pencil-tip-like, or line-linked contrast filling appearance are associated with higher chances of underlying ICAD on CTA or DSA. ${ }^{46,47}$

\section{Pretreatment}

In the $\mathrm{PCl}$ field for $\mathrm{CAD}$, pretreatment has been emphasized for reducing reocclusion and new vascular events with the use of DAP and high-dose statin serving as its pharmacological foundation. ${ }^{48,49}$ Although larger validation studies are still required, it seems reasonable to use the aforementioned baseline markers to identify LVOS potentially related to ICAD in anticipation to the intervention in order to plan the best pharmacological and mechanical treatment approaches. In this setting, prompt administration of DAP bolus prior to MT becomes a strong consideration if there is reasonable suspicion of underlying ICAD. As oral agents require a longer time to start acting, this is particularly applicable when there are expected delays in the treatment due to the need for inter-facility transfer or during off-hours when the neuroendovascular team is not readily available. Due to its faster onset of action and more reliable treatment response, ticagrelor $180 \mathrm{mg}$ oral bolus may be preferred to clopidogrel, ${ }^{50}$ although both drugs did not differ in outcomes when applied to extracranial stenting after $M{ }^{51}{ }^{51}$ The use of point-of-care testing in the angiography suite may help ensuring the patient is properly inhibited on the basis of oral agents alone in case stenting is needed.

Another important "pretreatment" decision in face of suspected underlying ICAD is whether or not to administer IVTs. As previously discussed, the use of antiplatelets in the setting of IVT remains controversial due to the theoretical increased hemorrhagic risks. At the same time, the rates of reocclusion following intravenous thrombolytics seem to be increased in the presence of ICAD. ${ }^{34}$ Meanwhile, three recent randomized trials have shown that the outcomes of MT alone did not differ those of intravenous alteplase followed by MT in patients presenting directly to thrombectomy capable centers (statistically significant for noninferiority in Direct Intraarterial Thrombectomy in Order to Revascularize Acute Ischemic Stroke Patients with Large Vessel Occlusion Efficiently in Chinese Tertiary Hospitals: a Multicenter Randomized Clinical Trial [DIRECT MT] and
Direct Endovascular Thrombectomy vs Combined IVT and Endovascular Thrombectomy for Patients With Acute Large Vessel Occlusion in the Anterior Circulation [DEVT] trials while insignificant in Direct Mechanical Thrombectomy in Acute LVO Stroke [SKIP] trial). ${ }^{52-54}$ These trials are from Asian countries where ICAD LVOS is relatively popular compared to embolic LVOS. As such, further studies should be done whether it is reasonable to skip IVT in patients suspected to have ICAD-related LVOS that can immediately undergo MT while IVT should still be given to all eligible patients presenting to non-thrombectomy capable centers. ${ }^{55}$

\section{Retrieval}

MT remains the only approved treatment method for intracranial LVOS. Initial thrombus debulking should, at least theoretically, decrease the chances of vessel reocclusion and/or distal embolization with angioplasty and/or stenting. However, it is worth mentioning that routine aspiration thrombectomy during PCl for ST-segment-elevation myocardial infarction has failed to improve both clinical outcomes and the rates of stent thrombosis; thus, the benefit of this approach in ICAD-related LVO still requires proper validation. ${ }^{56}$ Despite the underlying stenosis, it has been reported that superimposed in situ thrombus can be safely removed by using stent-retrievers. ${ }^{57,58}$ While MT by contact aspiration may represent another option, most studies have reported that stent retrieval as the first-line approach may lead to better results with higher rates of successful reperfusion and shorter reperfusion times as well as lower rates of rescue treatments and iatrogenic dissection or vessel rupture, and therefore should be considered as preferable initial option in most cases. ${ }^{59-61}$ There are a few potential explanations for the stent-retriever advantage in the setting of ICAD. The vessel is typically tapered in ICAD-related LVOS and this often prevents the direct contact between the aspiration catheter and the thrombus. Conversely, the stent-retriever can be more easily placed across the entire extent of the lesion and its active deployment may not only create a temporary bypass channel, that can provide some immediate relief to the ischemia and facilitate endogenous thrombolysis, but may also help delineating and characterizing the lesion enabling the planning of the next pharmacological (e.g., glycoprotein Ilb/IIla inhibitors [GPI] or cangrelor) and mechanical (sizing of angioplasty balloon and/or stent) strategies.

\section{Infusion of antiplatelets}

Even when initial recanalization can be achieved, intraprocedural reocclusion is highly likely in ICAD-related LVO. In a systematic review, reocclusion during MT occurred in 36.9\% of 
LVO patients with underlying ICAD versus $2.7 \%$ of those without it. ${ }^{62}$ Therefore, preventive strategies should be implemented immediately after the thrombus is debulked and underlying ICAD lesion is seen, whenever possible. However, MT is more frequently started in the absence of DAP because there is not a clear pretreatment suspicion for underlying ICAD, oral administration of drugs is not feasible, or there are concerns about the potential risks of hemorrhage due to extent of the infarct and/or use of intravenous thrombolytics. In this context, local or intravenous infusion of GPI has been associated with reduced reocclusion and improved clinical outcomes. ${ }^{3,63-65}$ The use of GPI is even more crucial in the setting of intracranial stenting given the even higher thrombogenicity associated with the addition of a foreign body. ${ }^{66}$ Indeed, most studies involving acute intracranial stenting for underlying ICAD reported that GPI was administered whenever feasible (Table 1). ${ }^{13-17}$ Although IV infusion may have a role as the bridge between the local infusion and oral antiplatelets, ${ }^{67}$ further studies are necessary to determine the optimal dose and duration after $\mathrm{EVT}$, with or without intracranial stenting. Notably, there is a good rationale to consider the use of acute antiplatelets even in ICAD-related LVO patients that have received IVT. As platelet-mediated thrombotic mechanisms are likely involved in rethrombosis after the thrombolytic-induced clot lysis, GPI may help maintaining vessel patency. ${ }^{34,68}$ Moreover, the CLEAR-Enhanced Regimen (CLEAR), CLEAR-Enhanced Regimen (CLEARER), and CLEAR-Full Dose Regimen (CLEAR-FDR) trials provide good safety data on the combination of eptifibatide plus alteplase at both reduced $(0.6 \mathrm{mg} / \mathrm{kg})$ and full dosages, granted that these studies are not specific to either LVO or MT patients. ${ }^{69}$ Finally, retrospective studies on the use of tirofiban during MT for ICAD-related LVO did not demonstrated any differences in the rates of sICH in patients who have versus not have received IVT. ${ }^{5,63,65}$ Notably, cangrelor, an intravenous P2Y12 receptor inhibitor with a rapid onset/offset of action and a short (3 to 6 minutes) half-life, may become a viable alternative to GPI in the near future. ${ }^{70,71}$ Once hemorrhagic complications have been excluded by post-procedural imaging, DAP should be started as soon as possible, especially when employing intracranial stenting.

\section{No-go decision}

Waiting and observing for reocclusion or further recanalization for about 10 to 20 minutes is reasonable prior to deciding whether to perform intracranial angioplasty and/or stenting. Sometimes, residual superimposed thrombus is further reduced by the endogenous lytic effect of flow restoration and the antithrombotic drugs during the observation time. Before reoc- clusion occurs, thrombus propagation or flow stagnation may be observed. If restenosis/recoiling or reocclusion occur despite GPI treatment, balloon angioplasty and/or stenting should then be considered.

\section{Treatment with angioplasty/stenting}

Prior to deciding on angioplasty/stenting, physicians should ponder that an adequate reperfusion is more important than a perfect recanalization. Clinical deterioration may paradoxically occur after technically improved recanalization with angioplasty/stenting as it may result in perforator occlusion or distal embolization. Therefore, proper patient selection is paramount and rescue therapy should be limited to situations of high reocclusion risk despite the use of antiplatelets.

In the following paragraphs, we describe the balloon angioplasty and stenting options and strategies. Although it is difficult to suggest any particular method, the best option can be decided based on the necessity of additional luminal and flow improvement following standard MT, collateral status, plaque extent and characteristics, the location of nearby perforators, and the risk of hemorrhagic complications.

\section{General treatment considerations}

The decision for rescue treatment is a major issue during the treatment of ICAD-related LVO. When deciding, the focus should be towards the best clinical rather than the best angiographic outcome. Based on this assumption, better decisions can be made thought careful analysis of the clinical and imaging data (Figure 3). First, the treating physicians should attempt to determine the pathophysiology of ICAD-related LVOS based on the infarct patterns, which can be classified as follows: (1) deep perforator infarcts, (2) small scattered infarcts, (3) borderzone infarcts, and (4) territorial infarcts. ${ }^{72}$ Deep perforator infarcts typically occur when a penetrating branch of the main trunk in a cerebral artery, such as middle cerebral artery (MCA) and basilar artery, is blocked and are not typically found in isolation in acute LVO cases. The blockage of deep perforators alone can result from propagation of in situ thrombi adjacent to the atherosclerotic lesions or from physical compression by the atherosclerotic plaque itself. This pattern cannot be improved by the means of any mechanical revascularization including balloon angioplasty or stenting. Small scattered infarcts can occur if multiple in situ thrombi embolize from the atherosclerotic lesion to distal vessels. This pattern is also not typically aided by angioplasty or stenting and, in the presence of adequate collateral flow with no or minimal clinical deficits can be attributed to established (versus ongoing) 


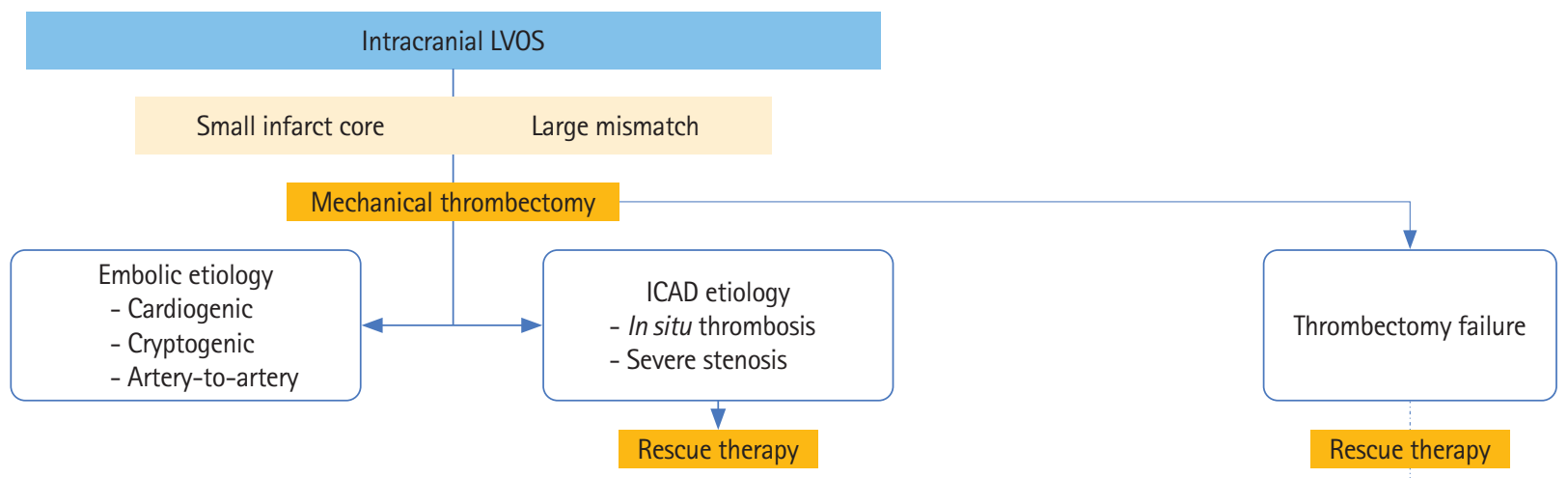

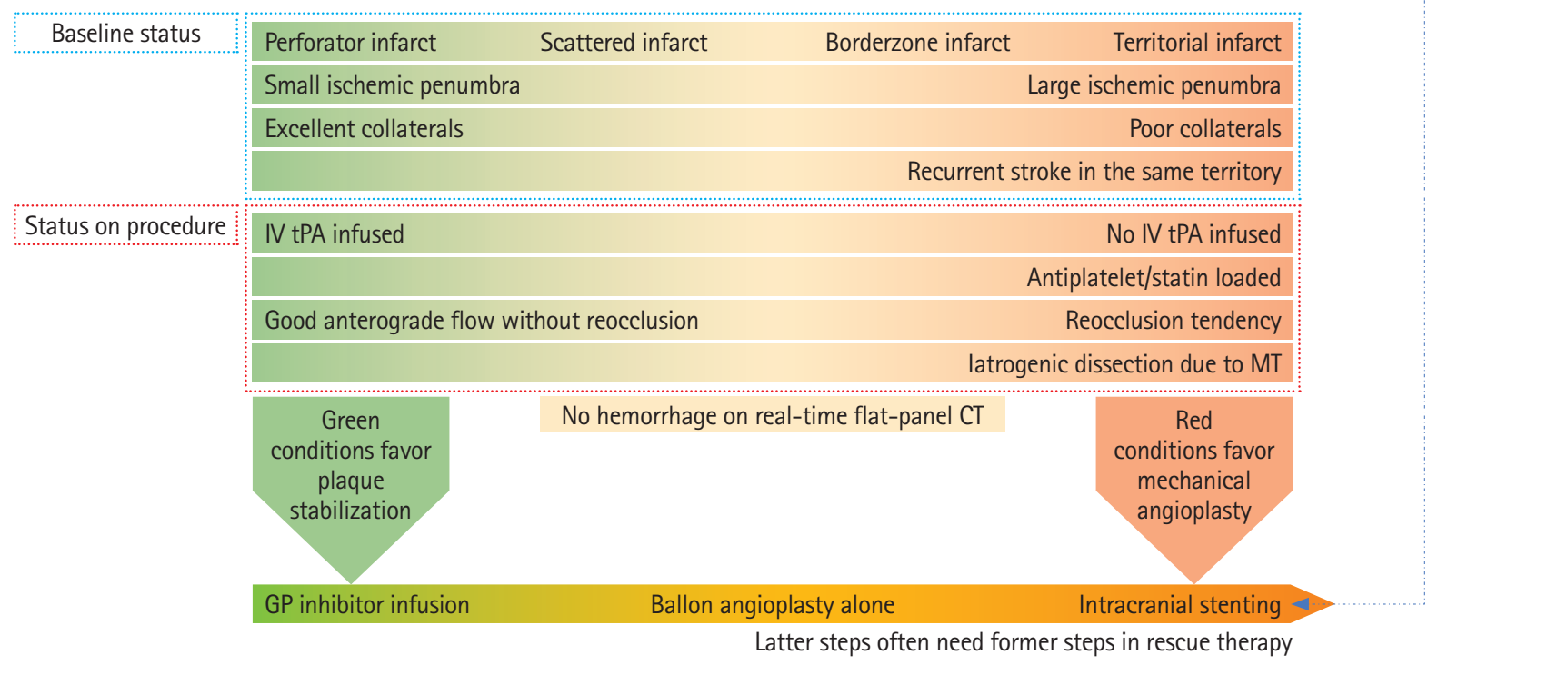

Figure 3. Considerations for rescue therapy in patients with intracranial atherosclerotic disease (ICAD)-related acute large vessel occlusion stroke (LVOS). Baseline and procedural conditions on the green area, which may include perforator infarct pattern, small ischemic penumbra, excellent collaterals, intravenous tissue plasminogen activator (IV tPA) infused, and good anterograde flow without reocclusion tendency after thrombectomy, favor glycoprotein (GP) infusion only. Conditions on the red area, which may include borderzone or territorial infarct pattern, large ischemic penumbra, poor collaterals, recurrent stroke due to the culprit ICAD lesion, no IV tPA infused, loading of antiplatelet or statin agents, reocclusion tendency after thrombectomy, and iatrogenic dissection due to thrombectomy, favor mechanical angioplasty. In case of thrombectomy failure, intracranial stenting has been reported to improve outcomes as rescue treatment. Intracranial stenting may necessitate GP inhibitor and balloon angioplasty as well. MT, mechanical thrombectomy.

ischemia, should be preferentially treated with medical therapy alone as a measure to prevent stump-emboli from the recently occluded vessel. Borderzone infarcts are typically caused hemodynamic compromise from severe stenosis or acute occlusion from in situ thrombosis, and as such, are likely to benefit from angioplasty and/or stenting. Territorial infarcts develop in the setting of even greater hemodynamic compromise with poor collaterals and large perfusion defects, which can be observed on baseline imaging; thus, rapid recanalization is even more critical. Second, baseline brain parenchymal images, revealing old infarcts, may provide additional considerations regarding the need for rescue therapy. If the stroke recurs in the same territory as ICAD, it might be related to preventive medication failure. In case of best medical treatment failure, the threshold for intracranial angioplasty/stenting during the emergent revascularization treatment can probably be lowered.
Third, the pretreatment pharmacological status is important. IVT infusion prior to EVT may make it difficult to decide on intracranial stenting because early administration of antiplatelet agents might be associated with an increased risk of intracerebral hemorrhage. ${ }^{35}$ In contrast, if sufficient antiplatelets have been on board prior to EVT, the additional risk of more aggressive antiplatelet treatment becomes a moot point and intracranial stenting would be more protected from reocclusion. Lastly, vessel status is another important consideration. The reocclusion tendency despite MT and/or GPI treatment is an important factor in the decision about the need for intracranial stenting. For this reason, physicians should consider waiting for 10 to 20 minutes before deciding to finish or perform further procedures. On the other hand, if plaque dissection is clearly seen after MT, intracranial stenting should be considered. Based on this background, we propose a tailored strategy for 
rescue treatments (Figure 3).

Between MT and rescue treatments, it is reasonable to check for the presence of intracerebral or subarachnoid hemorrhage on flat-panel CT prior to deciding about additional antiplatelet treatment and/or stent implantation. ${ }^{73}$ Moreover, the amount of parenchymal contrast hyperattenuation seen on the flat-panel CT has been reported to be associated with final infarct volume and clinical outcomes. ${ }^{74}$ This may add to the decision making by providing additional information about hemorrhagic risks and prognostication. Specifically, while we tend to favor rescue stenting for ICAD LVOS in the context of poor collaterals, small to moderate size territorial infarctions, and large perfusion defects, massive breakdown of the blood-brain barrier (as suggested by extensive parenchymal staining) may lead to hemorrhagic transformation and therefore permanent stent deployment should be avoided in this setting.

\section{ICAD lesion classification: technical and prognostic implications}

As for the results of balloon angioplasty and/or stenting for intracranial stenosis treatment, the technical outcomes are known to be highly associated with the characteristics of the atherosclerotic lesion. The classification of atherosclerotic lesions proposed by Mori et al. ${ }^{75}$ in 1998 showed a robust association of balloon angioplasty alone (BAA) with technical results. According to their report on balloon angioplasty for ICAS $>70 \%$, the lesions can be classified into three groups: type A, short ( $\leq 5 \mathrm{~mm}$ in length) concentric or moderately eccentric lesions less than totally occlusive; type $B$, tubular ( 5 to $10 \mathrm{~mm}$ in length) extremely eccentric or totally occluded lesions less

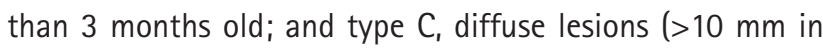
length), extremely angulated $\left(>90^{\circ}\right)$ lesions with excessive tortuosity of the proximal segment or totally occluded lesions 3 months old or older. While technical success rates were $92 \%$ and $86 \%$ in type $A$ and $B$ lesions, respectively, the rates were only $33 \%$ in type $C$ lesions. ${ }^{75}$ Moreover, the cumulative risks of fatal or nonfatal ischemic stroke or ipsilateral bypass surgery were $8 \%, 26 \%$, and $87 \%$ for type $A, B$, and C lesions, respectively. ${ }^{75}$ Therefore, balloon angioplasty for type $C$ lesions appears to be highly challenging. Although, these lesion types cannot be easily discriminated on baseline angiographic imaging in ICAD-related LVO, they may become recognizable during or after the use of MT devices.

More recently, Miao et al. ${ }^{76}$ reported on a tailored strategy of balloon angioplasty and/or stenting for symptomatic ICAS $>70 \%$ that was based on a modified Mori's classification. According to this strategy, patients were classified based on treatment devices: (1) balloon-mounted stent (BMS) group was applied for patients with smooth access and Mori type A lesions, or mid-basilar artery and distal M1 segment of MCA lesions; (2) balloon angioplasty plus self-expanding stent (SES) group for those with tortuous access and Mori type B or C lesions, or lesions with a significant mismatch in the diameter between the proximal and distal segment; and (3) BAA group for tortuous access and Mori type A lesions, or small target vessel diameter $(<2.5 \mathrm{~mm}) .^{76}$ In terms of technical success, the success rates were significantly different among the groups. The BMS and SES groups had success rates of $98 \%$ and $100 \%$, respectively, while it was only $90 \%$ in BAA group. ${ }^{76}$ Although the success rates were somewhat lower in the BAA group, each treatment strategy was assigned to mutually exclusive lesions so comparisons are not that meaningful. Nevertheless, overall, 96\% and at least 90\% of success rates are considerable improvements for symptomatic ICAS. In this successful treatment study, however, it was not obvious if the Mori type $C$ lesion was successfully treated by SES. Although both type B and C lesions were included in the SES group, the mean length of target lesion was relatively short compared to the BAA group. In another Chinese study, among 44 patients who were treated with an SES (Enterprise stent, Codman Neurovascular, Raynham, MA, USA), four patients had peri-procedural complications. Among them, three had type $C$ lesions reinforcing their poorer prognosis after angioplasty/stenting for ICAD.

Three-dimensional atherosclerotic burden with lesion length by stenotic degree is another prognostic factor. In a post hoc analysis from the SAMMPRIS trial, lesion length was an independent predictor of 30-day stroke or mortality in patients undergoing angioplasty and stenting. ${ }^{77}$ Likewise, pretreatment stenotic degree has been established as a strong predictor for in stent restenosis. ${ }^{78,79}$ Therefore, the combination of severe stenosis and long plaque length represents a significant challenge.

\section{Rescue mechanical treatment in ICAD-related LVOS}

\section{Emergent balloon angioplasty alone}

BAA has the advantage of greater simplicity when compared to angioplasty followed by SES placement and easier navigation when compared to BMS placement. Moreover, it might be the best choice when the optimal use of antithrombotic agents including GPI and DAP is not feasible due to increased concerns about the hemorrhagic risk, as the absence of stent implant makes the vascular environment theoretically less thrombogenic. However, BAA is associated with higher risks of arterial dissection potentially resulting in acute thrombosis or vessel 


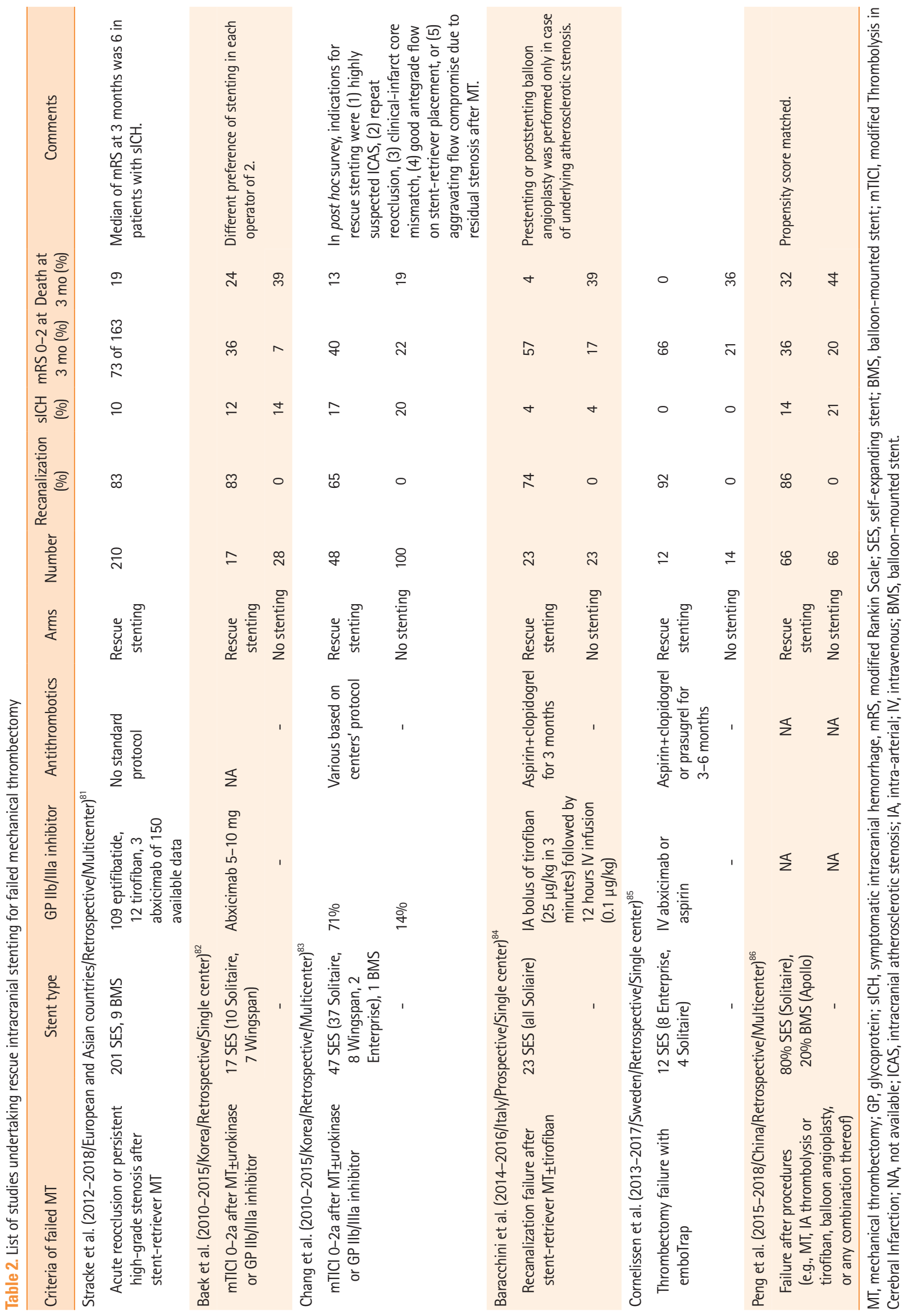


rupture, higher degree of residual stenosis due vessel recoiling, and higher vascular reocclusion due to wall hematomas. In this context, submaximal angioplasty by sizing the balloon to a nominal diameter at six atmospheres of $80 \%$ of the true luminal diameter (or approximately $60 \%$ in lesions directly adjacent to perforators) has been recommended to lower the risks of arterial dissection, vessel rupture and snowplowing. ${ }^{80}$

In face of the aforementioned limitations and the greater complexity of the atheromatous plaques typically encountered in acute LVO as compared to the "elective" treatment of stenotic lesions, we tend to reserve BAA for patients with short concentric lesions, especially when they have poor collaterals, which needs prompt recanalization, and when more aggressive antiplatelet treatment cannot be readily instituted.

\section{Emergent intracranial stenting}

Most studies regarding intracranial stenting for symptomatic ICAS have not been based on emergent situations, and therefore should not be translated into patients presenting with acute LVO. Nonetheless, rescue stenting after failed MT (presumably due to underlying ICAD in many of the cases) has been increasingly reported and has demonstrated promising results with successful recanalization in the $65 \%$ to $86 \%$ range (Table 2) ${ }^{81-86} \mathrm{~A}$ recent meta-analysis comprising four studies including a total of 352 patients who failed MT demonstrated that, as compared to non-stented failed MT patients ( $n=203)$, patients who were stent-rescued after failed MT $(n=149)$ had significantly higher rates of favorable clinical outcome (OR, $2.87 ; 95 \% \mathrm{Cl}, 1.77$ to $\left.4.66 ; P<0.001 ; I^{2}, 0 \%\right)$ and lower mortality $\left(\mathrm{OR}, 0.39 ; 95 \% \mathrm{Cl}, 0.16\right.$ to $\left.0.93 ; P=0.03 ; I^{2}, 43 \%\right)$ at 90 days, without any increased chances of $\mathrm{sICH}(\mathrm{OR}, 0.68 ; 95 \% \mathrm{Cl}, 0.37$ to $\left.1.27 ; P=0.23 ; I^{2}, 0 \%\right){ }^{87} \mathrm{~A}$ multicenter retrospective series including 210 patients treated with rescue stenting after failed stent-retriever MT showed rates of good functional outcome and $\mathrm{sICH}$ of $44.8 \%$ and $10.5 \%$, respectively. ${ }^{16} \mathrm{~A}$ higher admission National Institutes of Health Stroke Scale (NIHSS) (adjusted $O R, 1.10 ; P=0.002$ ), a higher premorbid mRS (adjusted $O R$, 2.02; $P=0.049$ ), and failed reperfusion after stenting (modified Thrombolysis in Cerebral Infarction [mTICl] 0-2a, adjusted OR, 23.24; $P<0.001$ ) were independent predictors of poor functional outcome. ${ }^{81}$ These studies reinforce the critical importance of reperfusion in LVOS and suggest that angioplasty/stenting should be promptly considered as a rescue option.

\section{Considerations in stent selection}

Which stent should be selected is next question after intracranial stenting is decided for rescue treatment. Although various situations are encountered during EVT in patients with
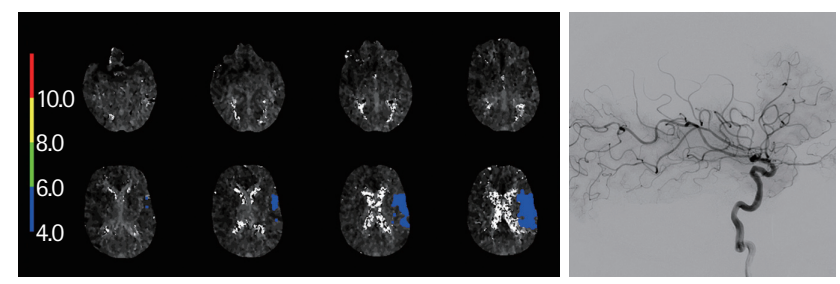

A

B
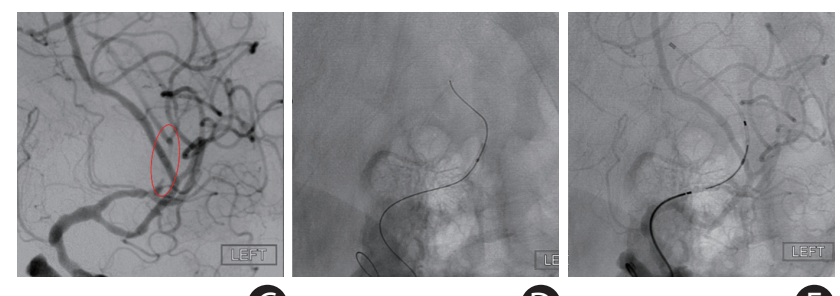

C

(D)
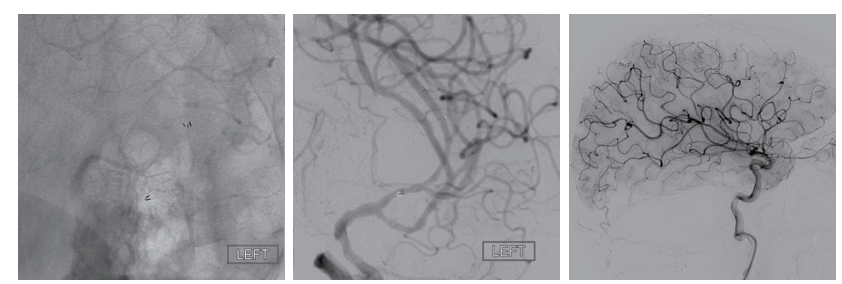

$\boldsymbol{F}$

G

$\boldsymbol{\Theta}$

Figure 4. A self-expanding stent (SES) placement for small vessel diameter around culprit stenosis. A 77-year-old African-American female with hypertension, hyperlipidemia, and diabetes mellitus had acute steno-occlusive intracranial atherosclerotic disease (ICAD) and presented with an unwitnessed fall the night prior. In the following morning, she presented to an outside hospital with aphasia and right hemiparesis. After telemedicine consultation, she was loaded with clopidogrel $600 \mathrm{mg}+$ aspirin $325 \mathrm{mg}$ and transferred for potential endovascular intervention. Upon arrival, National Institutes of Health Stroke Scale was 14, and non-contrast computed tomography (CT) showed chronic scattered borderzone infarcts in the left hemisphere. (A) CT perfusion showed a delay on Tmax $>4$ seconds in the left middle cerebral artery superior division territory. She was brought to angiography suite for endovascular reperfusion treatment. VerifyNow, a point-of-care testing, showed acceptable platelet inhibition (PRU 210). (B) Initial digital subtraction angiography showed diffuse ICAD with severe stenosis of a left insular M3 branch resulting in critical hypoperfusion. (C) The atherosclerotic lesion was long $(>10 \mathrm{~mm})$ and located is a small vessel $(1.5 \mathrm{~mm})$ along a curved course, so a SES was chosen for intracranial stenting. (D) Balloon angioplasty was performed (Sprinter Legend Rx angioplasty balloon, $1.5 \times 12 \mathrm{~mm}$ ) over an exchange-length microguidewire. The balloon catheter was exchanged for a 0.021 microcatheter. (E) An Enterprise-2 stent $(4 \times 23 \mathrm{~mm})$ was advanced over the microcatheter. (F) The stent was unsheathed and deployed. (G) The vessel was successfully recanalized. (H) Final angiography showed complete recanalization and reperfusion. She made a complete functional recovery.

ICAD-related LVOS, the number of approved devices for intracranial neurovascular stent remains limited and many coronary devices are still routinely used off-label. Intracranial stents can be largely classified into SES and BMS. Among SES, aneurysm-bridging intracranial SES including the Solitaire (Medtronic Neurovascular, Irvine, CA, USA), Enterprise, and Neuroform (Stryker, Kalamazoo, MI, USA) has been widely used 
off-label in ICAD-related acute LVOS. The Solitaire AB, originally developed for assisting coil embolization, shares a very similar design to the Solitaire stent-retrievers approved for MT with the distinguishing feature of a controlled detachment option. As such, Solitaire stents have been reported to be deployed in patients with recanalization failure by MT, with or without underlying ICAD, ${ }^{82,83}$ as well as in elective cases of complex symptomatic intracranial stenosis. ${ }^{88}$ The Enterprise stent has also been used for a relatively long time in patients with symptomatic intracranial stenosis. ${ }^{79,89-93}$ Its radial force is known to be moderate, making navigation and advancement relatively easier and a good option for long lesions in a tortuous anatomy (an example case in Figure 4). The Neuroform EZ stent has also been used for symptomatic intracranial stenosis but has been now largely replaced by the newer-generation low-profile Neuroform Atlas. ${ }^{94,95}$

Both Wingspan/Gateway (Stryker, Kalamazoo, MI, USA) and Credo SES/NeuroSpeed PTA Balloon Catheter (Acandis GmbH, Pforzheim, Germany) systems are CE mark. Notably, neither system has been approved for acute use in ICAD-related LVO. Although the Wingspan SES and the Gateway PTA Balloon Catheter are the only U.S. Food and Drug Administra- tion-cleared devices for symptomatic ICAS, many interventionists prefer the other systems over the more cumbersome and outdated delivery system of the Wingspan, which in the setting of acute LVOS is equally off-label and unproven. The Credo/ NeuroSpeed system employs a novel concept where the stent can be delivery through the actual balloon catheter immediately following the angioplasty. This obviates the need for over-the-wire exchanges, one of the most technically demanding and risky steps associated with the placement of SES. Notably, we have been able to perform the exact same technique through the off-label use of small over-the-wire coronary angioplasty balloon catheters (Mini Trek OTW, Abbott Laboratories, Abbott Park, IL, USA) and low-profile aneurysm-remodeling SES (Neuroform Atlas, Kalamazoo, MI, USA) (an example case in Figure 5).

Coronary BMS are commonly used to treat ICAD. BMS are harder to navigate because the addition of the mounted balloon decreases the system flexibility making it more difficult to advance through tortuous anatomy, such as the ICA siphon or the carotid terminus. Nevertheless, when technically feasible, it is regarded that the use of BMS is usually easier and faster than SES because the balloon angioplasty and deployment of

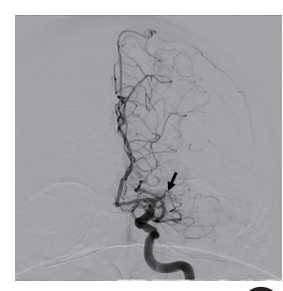

A

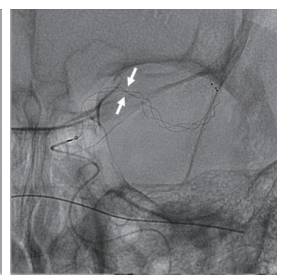

B

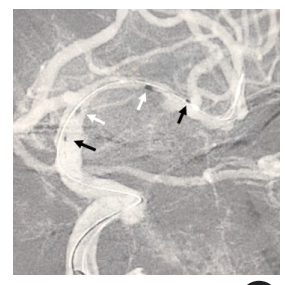

(E)

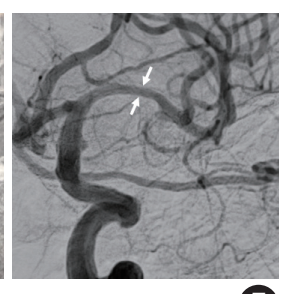

(
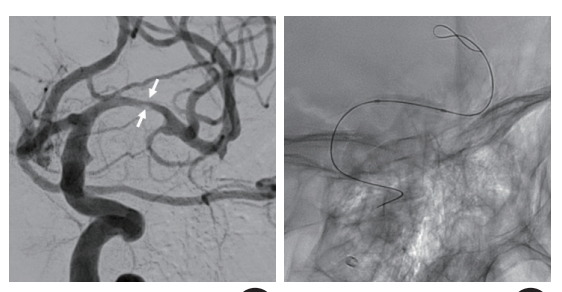

C

(D)

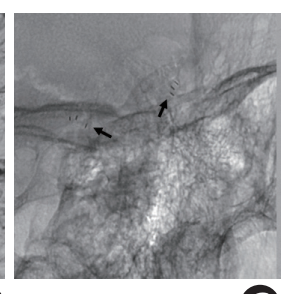

$\boldsymbol{G}$

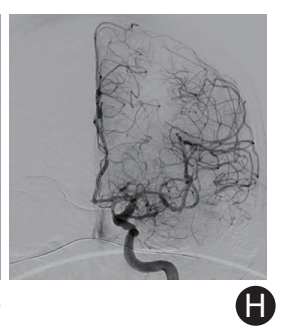

Figure 5. A self-expanding stent (SES) placement for landing zone tortuosity and mismatch: directly after intracranial balloon angioplasty (without exchange of the balloon catheter) for acute occlusion in the M1 segment of the left middle cerebral artery (MCA) due to underlying intracranial atherosclerotic disease in 54-year-old African-American male with history of diabetes mellitus and prior stroke in left MCA presented to the emergency room with aphasia and mild right hemiparesis (National Institutes of Health Stroke Scale 6). (A) Computed tomography angiography (not shown) and conventional angiography showed complete occlusion of the left MCA-M1 (black arrow) with evidence of multifocal intracranial stenosis. (B) Mechanical thrombectomy was performed with a Trevo XP (4×30 mm) stent-retriever. There was severe "pinching" of the device suggesting underlying intracranial atherosclerotic disease (white arrows) versus "hard-clot." (C) A focal severe stenosis (white arrows) was disclosed after one device pass. There was a mismatch across the diameters of stent landing zones due to the presence of post-stenotic dilation and a trifurcation, thus a SES was selected for intracranial stenting. Intravenous tirofiban bolus was administered in anticipation to the stent implantation. (D) Balloon was performed using a Mini Trek $2 \times 12 \mathrm{~mm}$ over-the-wire coronary balloon catheter. Subsequently, the balloon catheter was advanced 2 to $3 \mathrm{~mm}$ and the microwire was removed. (E) A Neuroform Atlas $4.5 \times 22 \mathrm{~mm}$ SES was then advanced through the coronary balloon catheter and placed across the target lesion. The stent was then carefully unsheathed by withdrawing the balloon catheter (black arrows, distal and proximal ends of the stent; white arrows distal and proximal ends of the balloon catheter during stent deployment). (F, G) There was complete resolution of the stenosis (white arrows) with good wall apposition (black arrows). (H) Final angiography shows complete recanalization and reperfusion. The patient recovered to his baseline. 

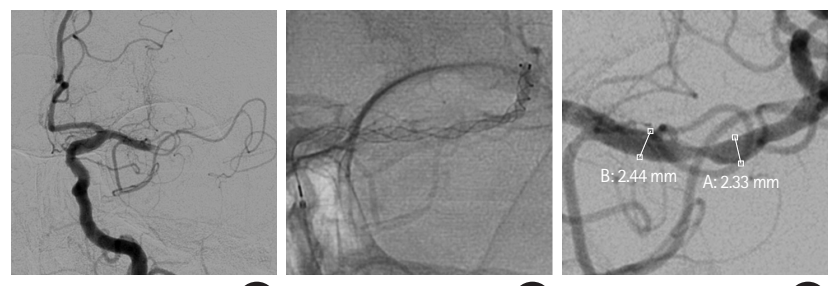

A

B
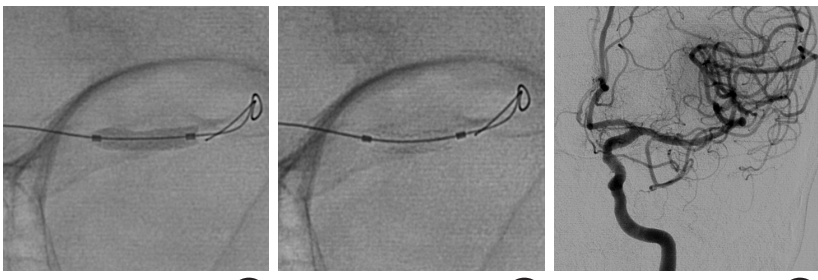

D

E

$\boldsymbol{\theta}$

Figure 6. Placement of a balloon-mounted stent (BMS) for large vessel diameter and short length of the culprit stenosis. An acute middle cerebral artery (MCA) occlusion occurred in a 79-year-old female with history of previous stroke due to stenosis in M1 segment of left MCA (baseline modified Rankin Scale 1) currently treated with aspirin monotherapy presenting to an outside hospital with fluctuating severe aphasia and right hemiparesis. After telemedicine consultation, she was loaded with ticagrelor $180 \mathrm{mg}$ prior to transfer. Upon arrival, National Institutes of Health Stroke Scale was 13 and time from last known normal was greater than 12 hours. Multimodal computed tomography (CT) showed left MCA-M1 occlusion with Alberta Stroke Program Early CT Score 9 and a large perfusion mismatch. The patient was brought to angiography suite for endovascular reperfusion treatment. (A) Initial angiogram showed complete left M1 occlusion. (B) Standard thrombectomy was performed with a Trevo XP $(4 \times 30 \mathrm{~mm})$ stent-retriever. (C) After one device pass, a focal severe stenosis was found. The atherosclerotic lesion was short, and both diameters of stent landing zones were similar and over $2 \mathrm{~mm}$, so a BMS was chosen for intracranial stenting. (D) The mounted balloon is inflated for deployment. (E) The Integrity stent $(2.25 \times 9 \mathrm{~mm})$ is deployed with good wall apposition. (F) Final angiography shows complete recanalization and reperfusion. The patient recovered to her baseline.

the stent are both part of a single step (an example case in Figure 6). ${ }^{76}$ The navigation difficulties can be typically overcome by selecting the shortest possible stent that can treat the lesion and then delivering it through a distal intermediate catheter. These catheters are highly navigable and can nearly invariably be placed just proximally to the stenosis in anticipation to the BMS navigation. The Apollo BMS (MicroPort Medical, Shanghai, China) is approved for intracranial use in China. A study involving 159 symptomatic ICAS patients treated with the Apollo BMS demonstrated a technical success of $98.7 \%$ with $4.4 \%$ rate of stroke, transient ischemic attack, or death at 30-day follow-up. ${ }^{96}$ Our largest personal experience in ICAD-related LVO has been with the Integrity Rx BMS (Medtronic, Minneapolis, MN, USA) but we have more recently switched to use the Resolute Onyx Zotarolimus-Eluting Stent (Medtronic) due to its improved flexibility and conformability, and due to the availability of a $2 \mathrm{~mm}$ diameter, which makes it highly suitable
Balloon-mounted stents

$\geq 2-2.25 \mathrm{~mm}$

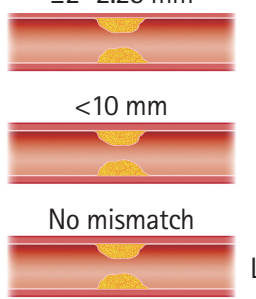

No

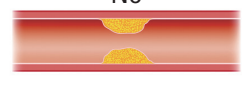

Landing zone tortuosity

Figure 7. Indications of self-expanding stents vs. balloon-mounted stents for underlying intracranial atherosclerotic disease in acute large vessel occlusion stroke.

to MCA lesions. The Resolute Onyx Zotarolimus delivery inhibits neointimal growth and thus may reduce the incidence of long-term stenosis. The stent platform also includes a biocompatible polymer that may contribute to reduce inflammation and thrombosis. A recent single center study encompassing 18 consecutive patients undergoing elective treatment for symptomatic ICAS demonstrated 100\% technical success, and no reported periprocedural strokes or death. ${ }^{97}$

There are key anatomic factors that should be considered when choosing SES versus BMS as the most appropriate device to rescue the underlying ICAS, including: (1) vessel diameter, (2) lesion length, (3) vessel diameter mismatch, and (4) vessel tortuosity (Figure 7). Since the smallest currently available BMS dimeter is $2 \mathrm{~mm}$, SES are the safest option whenever the vessel diameter at the target lesion is less than $2 \mathrm{~mm}$ whereas both options are acceptable for the vessel diameter $\geq 2 \mathrm{~mm}$. We prefer to reserve the use of BMS for shorter lesion lengths as the navigation of long BMS can be difficult. Alternatively, a longer lesion can be covered by two telescoping BMS sequentially deployed from distal to proximal but we generally favor SES for the longer lesions and even more so in the presence of tortuosity. Likewise, SES are preferable whenever there is a significant difference in the proximal and distal diameters of the lesion. Finally, if the landing zone is tortuous, SES might be the best choice since they are not only more navigable but also have better conformability. Given the simpler technique, short procedural time and lower cost associated with BMS, they tend to be our first option whenever technically feasible with SES being reserved for the remaining cases.

\section{Future perspectives}

There are two ongoing studies aiming to evaluate the safety 
and efficacy of rescue intracranial angioplasty/stenting in acute LVOS patients who have failed standard MT. The Acandis Credo Intracranial Stent for Unsuccessful Recanalization After Thrombectomy (ACUTE) trial (ClinicalTrials.gov, NCT03955835) is a prospective, single-arm, open-label, multicenter trial including patients with intracranial ICA or MCA occlusion with unsuccessful recanalization after MT and suspected underlying stenosis amenable to stenting with the Credo/NeuroSpeed system. The Rescue Stenting for Failed Endovascular Thrombectomy in Acute Ischemic Stroke (ReSET) study (ClinicalTrials.gov, NCT03993340) is a prospective, open-label, multicenter registry of rescue intracranial stenting with detachment of the Solitaire device in patients with acute occlusions of the intracranial ICA or MCA-M1 who have failed MT.

Recanalization failure may also occur in other settings than ICAD including refractory fibrin-rich or calcific emboli. The results of the aforementioned studies will hopefully provide pragmatic data regarding the implications for intracranial stenting in acute ICAD and other more refractory forms of LVOS. For a clinical trial, randomization is challenging due to the lack of standardized diagnostic methods prior to MT. Currently, a number of researchers are studying the baseline markers of underlying ICAD in acute LVOS, and this will hopefully allow for the planning of future studies specifically targeting the ICAD population.

\section{Conclusions}

The treatment of patients with ICAD-related acute LVO is challenging. Nevertheless, a meticulous approach including optimal patient selection, pathophysiology-based decisions, and a rational choice of drug and devices seems to result in improved clinical outcomes. Proper validation of the best pharmacological and mechanical strategies through prospective randomized clinical trials are warranted.

\section{Disclosure}

Raul G. Nogueira reports consulting fees for advisory roles with Anaconda, Biogen, Cerenovus, Genentech, Hybernia, Imperative Care, Medtronic, Phenox, Philips, Prolong Pharmaceuticals, Stryker Neurovascular, Shanghai Wallaby, Synchron, and stock options for advisory roles with Astrocyte, Brainomix, Cerebrotech, Ceretrieve, Corindus Vascular Robotics, Vesalio, Viz-Al, RapidPulse, and Perfuze. Raul G. Nogueira is one of the Principal Investigators of the "Endovascular Therapy for Low NIHSS Ischemic Strokes (ENDOLOW)" trial. Funding for this project is provided by Cerenovus. Raul G. Nogueira is an investor in Viz-Al,
Perfuze, Cerebrotech, Reist/Q'Apel Medical, Truvic, and Viseon.

\section{Acknowledgments}

Special thanks to Hyoung-Mo Yang, a professor in Department of Cardiology, Ajou University School of Medicine, who gave his expert opinions on this article, and Hyeon-Min Lee, a researcher in Department of Neurology, who illustrated schematic figures.

\section{References}

1. Powers WJ, Rabinstein AA, Ackerson T, Adeoye OM, Bambakidis NC, Becker $\mathrm{K}$, et al. Guidelines for the early management of patients with acute ischemic stroke: 2019 update to the 2018 guidelines for the early management of acute ischemic stroke. A guideline for healthcare professionals from the American Heart Association/American Stroke Association. Stroke 2019;50:e344-e418.

2. Ko SB, Park HK, Kim BM, Heo JH, Rha JH, Kwon SU, et al. 2019 Update of the Korean clinical practice guidelines of stroke for endovascular recanalization therapy in patients with acute ischemic stroke. J Stroke 2019;21:231-240.

3. Baik SK, Oh SJ, Park KP, Lee JH. Intra-arterial tirofiban infusion for partial recanalization with stagnant flow in hyperacute cerebral ischemic stroke. Interv Neuroradiol 2011;17: 442-451.

4. Kang DH, Yoon W, Kim SK, Baek BH, Lee YY, Kim YW, et al. Endovascular treatment for emergent large vessel occlusion due to severe intracranial atherosclerotic stenosis. J Neurosurg 2019;130:1949-1956.

5. Baek JH, Kim BM, Heo JH, Kim DJ, Nam HS, Kim YD. Outcomes of endovascular treatment for acute intracranial atherosclerosis-related large vessel occlusion. Stroke 2018; 49:2699-2705.

6. Lee JS, Hong JM, Lee KS, Suh HI, Demchuk AM, Hwang YH, et al. Endovascular therapy of cerebral arterial occlusions: intracranial atherosclerosis versus embolism. J Stroke Cerebrovasc Dis 2015;24:2074-2080.

7. Jia B, Feng L, Liebeskind DS, Huo X, Gao F, Ma N, et al. Mechanical thrombectomy and rescue therapy for intracranial large artery occlusion with underlying atherosclerosis. J Neurointerv Surg 2018;10:746-750.

8. Dobrocky T, Kaesmacher J, Bellwald S, Piechowiak E, Mosimann PJ, Zibold F, et al. Stent-retriever thrombectomy and rescue treatment of M1 occlusions due to underlying intracranial atherosclerotic stenosis: cohort analysis and review of the literature. Cardiovasc Intervent Radio/ 2019;42:863-872. 
9. Kim YW, Hong JM, Park DG, Choi JW, Kang DH, Kim YS, et al. Effect of intracranial atherosclerotic disease on endovascular treatment for patients with acute vertebrobasilar occlusion. AJNR Am J Neuroradiol 2016;37:2072-2078.

10. Lee JS, Lee SJ, Yoo JS, Hong JH, Kim CH, Kim YW, et al. Prognosis of acute intracranial atherosclerosis-related occlusion after endovascular treatment. J Stroke 2018;20:394-403.

11. Turan TN, Cotsonis G, Lynn MJ, Wooley RH, Swanson S, Wil-

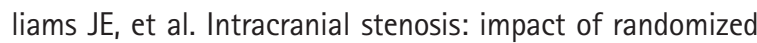
trials on treatment preferences of US neurologists and neurointerventionists. Cerebrovasc Dis 2014;37:203-211.

12. Zaidat 00, Castonguay AC, Nguyen TN, Becker KJ, Derdeyn $\mathrm{CP}$, Nelson PK, et al. Impact of SAMMPRIS on the future of intracranial atherosclerotic disease management: polling results from the ICAD symposium at the International Stroke Conference. J Neurointerv Surg 2014;6:225-230.

13. Kim JG, Suh DC, Song Y, Choi JC, Lee DH. Direct stenting of intracranial atherosclerosis-related acute large vessel occlusion. Clin Neuroradiol 2021;31:833-841.

14. Wu C, Chang W, Wu D, Wen C, Zhang J, Xu R, et al. Angioplasty and/or stenting after thrombectomy in patients with underlying intracranial atherosclerotic stenosis. Neuroradiology 2019;61:1073-1081.

15. Gross BA, Desai SM, Walker $G$, Jankowitz BT, Jadhav $A$, Jovin TG. Balloon-mounted stents for acute intracranial large vessel occlusion secondary to presumed atherosclerotic disease: evolution in an era of supple intermediate catheters. $J \mathrm{Neu}$ rointerv Surg 2019;11:975-978.

16. Feng MT, Zhang HJ, Zhang $Y X$, Xing PF, Zhang L, Zhang YW, et al. Stent angioplasty for acute intracranial atherosclerotic occlusion after failed thrombectomy: a single-institution series of 55 patients. World Neurosurg 2019;130:e444-e448.

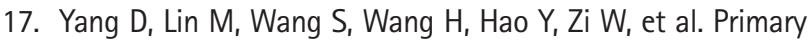
angioplasty and stenting may be superior to thrombectomy for acute atherosclerotic large-artery occlusion. Interv Neuroradiol 2018;24:412-420.

18. Yoon W, Kim SK, Park MS, Kim BC, Kang HK. Endovascular treatment and the outcomes of atherosclerotic intracranial stenosis in patients with hyperacute stroke. Neurosurgery 2015;76:680-686.

19. Rai $A T$, Seldon $A E$, Boo $S$, Link PS, Domico JR, Tarabishy AR, et al. A population-based incidence of acute large vessel occlusions and thrombectomy eligible patients indicates significant potential for growth of endovascular stroke therapy in the USA. J Neurointerv Surg 2017;9:722-726.

20. Kim JS, Kim YJ, Ahn SH, Kim BJ. Location of cerebral atherosclerosis: why is there a difference between East and West? Int J Stroke 2018;13:35-46.
21. Lee D, Lee DH, Suh DC, Kim BJ, Kwon SU, Kwon HS, et al. Endovascular treatment in patients with cerebral artery occlusion of three different etiologies. J Stroke 2020;22:234-244.

22. Suh $\mathrm{HI}$, Hong JM, Lee KS, Han M, Choi JW, Kim JS, et al. Imaging predictors for atherosclerosis-related intracranial large artery occlusions in acute anterior circulation stroke. J Stroke 2016;18:352-354.

23. Baek JH, Kim BM. Angiographical identification of intracranial, atherosclerosis-related, large vessel occlusion in endovascular treatment. Front Neurol 2019;10:298.

24. Chimowitz MI, Lynn MJ, Derdeyn CP, Turan TN, Fiorella D, Lane $B F$, et al. Stenting versus aggressive medical therapy for intracranial arterial stenosis. N Engl J Med 2011;365:9931003.

25. Zaidat 00, Fitzsimmons BF, Woodward BK, Wang Z, Killer-Oberpfalzer $M$, Wakhloo $A$, et al. Effect of a balloon-expandable intracranial stent vs medical therapy on risk of stroke in patients with symptomatic intracranial stenosis: the VISSIT randomized clinical trial. JAMA 2015;313:12401248.

26. Libby P. Inflammation in atherosclerosis. Nature 2002;420: 868-874.

27. van der Wal AC. Coronary artery pathology. Heart 2007;93: 1484-1489.

28. Guidelines for the performance of percutaneous transluminal coronary angioplasty. Circulation 1982;66:693-694.

29. Iqbal J, Gunn J, Serruys PW. Coronary stents: historical development, current status and future directions. Br Med Bull 2013;106:193-211.

30. Levine GN, Bates ER, Blankenship JC, Bailey SR, Bittl JA, Cercek $B$, et al. 2011 ACCF/AHA/SCAl guideline for percutaneous coronary intervention: a report of the American College of Cardiology Foundation/American Heart Association task force on practice guidelines and the Society for Cardiovascular Angiography and Interventions. Circulation 2011;124: e574-e651.

31. Lee RM. Morphology of cerebral arteries. Pharmacol Ther 1995;66:149-73.

32. Fiorella D, Derdeyn CP, Lynn MJ, Barnwell SL, Hoh BL, Levy El, et al. Detailed analysis of periprocedural strokes in patients undergoing intracranial stenting in Stenting and Aggressive Medical Management for Preventing Recurrent Stroke in Intracranial Stenosis (SAMMPRIS). Stroke 2012;43:2682-2688.

33. Khatri R, McKinney AM, Swenson B, Janardhan V. Bloodbrain barrier, reperfusion injury, and hemorrhagic transformation in acute ischemic stroke. Neurology 2012;79(13 Suppl 1):S52-S57.

34. Heo JH, Lee KY, Kim SH, Kim DI. Immediate reocclusion fol- 
lowing a successful thrombolysis in acute stroke: a pilot study. Neurology 2003;60:1684-1687.

35. Zinkstok SM, Beenen LF, Majoie CB, Marquering HA, de Haan $\mathrm{RJ}$, Roos YB. Early deterioration after thrombolysis plus aspirin in acute stroke: a post hoc analysis of the Antiplatelet Therapy in Combination with Recombinant t-PA Thrombolysis in Ischemic Stroke trial. Stroke 2014;45:3080-3082.

36. Cappellari M, Turcato G, Forlivesi S, Zivelonghi C, Bovi P, Bonetti $B$, et al. STARTING-SICH nomogram to predict symptomatic intracerebral hemorrhage after intravenous thrombolysis for stroke. Stroke 2018;49:397-404.

37. Tsivgoulis G, Katsanos AH, Mavridis D, Gdovinova Z, Karliński $M$, Macleod MJ, et al. Intravenous thrombolysis for ischemic stroke patients on dual antiplatelets. Ann Neurol 2018;84: 89-97.

38. Altersberger VL, Sturzenegger R, Räty $S$, Hametner $C$, Scheitz $J F$, Moulin $S$, et al. Prior dual antiplatelet therapy and thrombolysis in acute stroke. Ann Neurol 2020;88:857-859.

39. Adams HP Jr, Bendixen BH, Kappelle $\sqcup$, Biller J, Love BB, Gordon DL, et al. Classification of subtype of acute ischemic stroke. Definitions for use in a multicenter clinical trial. TOAST. Trial of Org 10172 in Acute Stroke Treatment. Stroke 1993;24:35-41.

40. Tsang A, Lau KK, Tsang F, Tse M, Lee R, Lui WM. Severity of intracranial carotid artery calcification in intracranial atherosclerosis-related occlusion treated with endovascular thrombectomy. Clin Neurol Neurosurg 2018;174:214-216.

41. Baek JH, Kim BM, Kim JW, Kim DJ, Heo JH, Nam HS, et al. Utility of leptomeningeal collaterals in predicting intracranial atherosclerosis-related large vessel occlusion in endovascular treatment. J Clin Med 2020;9:2784.

42. Baek JH, Kim BM, Kim DJ, Heo JH, Nam HS, Song D, et al. Importance of truncal-type occlusion in stentriever-based thrombectomy for acute stroke. Neurology 2016;87:15421550.

43. Baek JH, Kim BM, Yoo J, Nam HS, Kim YD, Kim DJ, et al. Predictive value of computed tomography angiography-determined occlusion type in stent retriever thrombectomy. Stroke 2017:48:2746-2752.

44. Lee SJ, Hong JM, Choi JW, Kang DH, Kim YW, Kim YS, et al. CTA-based truncal-type occlusion is best matched with postprocedural fixed focal stenosis in vertebrobasilar occlusions. Front Neuro/ 2019;9:1195.

45. Haussen DC, Bouslama M, Dehkharghani S, Grossberg JA, Bianchi $N$, Bowen $M$, et al. Automated CT perfusion prediction of large vessel acute stroke from intracranial atherosclerotic disease. Interv Neurol 2018;7:334-340.

46. Garcia-Bermejo P, Patro SN, Ahmed AZ, Al Rumaihi G, Akhtar
$\mathrm{N}$, Kamran S, et al. Baseline occlusion angiographic appearance on mechanical thrombectomy suggests underlying etiology and outcome. Front Neurol 2019;10:499.

47. Jin $X$, Shi $F$, Chen $Y$, Zheng $X$, Zhang J. Jet-like appearance in angiography as a predictive image marker for the occlusion of intracranial atherosclerotic stenosis. Front Neurol 2020; $11: 575567$.

48. Urban P, Macaya C, Rupprecht HJ, Kiemeneij F, Emanuelsson $H$, Fontanelli $A$, et al. Randomized evaluation of anticoagulation versus antiplatelet therapy after coronary stent implantation in high-risk patients: the multicenter aspirin and ticlopidine trial after intracoronary stenting (MATIS). Circulation 1998;98:2126-2132.

49. Patti G, Pasceri V, Colonna G, Miglionico M, Fischetti D, Sardella $\mathrm{G}$, et al. Atorvastatin pretreatment improves outcomes in patients with acute coronary syndromes undergoing early percutaneous coronary intervention: results of the ARMYDA-ACS randomized trial. J Am Coll Cardiol 2007;49: 1272-1278.

50. Husted S, Emanuelsson H, Heptinstall S, Sandset PM, Wickens $M$, Peters $G$. Pharmacodynamics, pharmacokinetics, and safety of the oral reversible P2Y12 antagonist AZD6140 with aspirin in patients with atherosclerosis: a double-blind comparison to clopidogrel with aspirin. Eur Heart J 2006;27: 1038-1047.

51. Bücke $P$, Aguilar Pérez $M$, AlMatter $M$, Hellstern V, Bäzner $H$, Henkes $\mathrm{H}$. Functional outcome and safety of intracranial thrombectomy after emergent extracranial stenting in acute ischemic stroke due to tandem occlusions. Front Neurol 2018;9:940.

52. Yang $P$, Zhang $Y$, Zhang $L$, Zhang $Y$, Treurniet KM, Chen $W$, et al. Endovascular thrombectomy with or without intravenous alteplase in acute stroke. N Engl J Med 2020;382:1981-1993.

53. Suzuki K, Matsumaru Y, Takeuchi M, Morimoto M, Kanazawa $R$, Takayama $Y$, et al. Effect of mechanical thrombectomy without vs with intravenous thrombolysis on functional outcome among patients with acute ischemic stroke: the SKIP randomized clinical trial. JAMA 2021;325:244-253.

54. Zi W, Qiu Z, Li F, Sang H, Wu D, Luo W, et al. Effect of endovascular treatment alone vs intravenous alteplase plus endovascular treatment on functional independence in patients with acute ischemic stroke: the DEVT randomized clinical trial. JAMA 2021;325:234-243.

55. Nogueira RG, Tsivgoulis G. Large vessel occlusion strokes after the DIRECT-MT and SKIP trials: is the alteplase syringe half empty or half full? Stroke 2020;51:3182-3186.

56. Jolly SS, James S, Džavík V, Cairns JA, Mahmoud KD, Zijlstra $F_{1}$ et al. Thrombus aspiration in ST-segment-elevation myo- 
cardial infarction: an individual patient meta-analysis. Thrombectomy Trialists Collaboration. Circulation 2017;135: 143-152.

57. Lee JS, Hong JM, Lee KS, Suh HI, Choi JW, Kim SY. Primary stent retrieval for acute intracranial large artery occlusion due to atherosclerotic disease. J Stroke 2016;18:96-101.

58. Lee JS, Lee SJ, Hong JM, Choi JW, Yoo J, Hong JH, et al. Solitaire thrombectomy for acute stroke due to intracranial atherosclerosis-related occlusion: ROSE ASSIST study. Front Neurol 2018;9:1064.

59. Yoo J, Lee SJ, Hong JH, Kim YW, Hong JM, Kim CH, et al. Immediate effects of first-line thrombectomy devices for intracranial atherosclerosis-related occlusion: stent retriever versus contact aspiration. BMC Neurol 2020;20:283.

60. Kang DH, Yoon W, Baek BH, Kim SK, Lee YY, Kim JT, et al. Front-line thrombectomy for acute large-vessel occlusion with underlying severe intracranial stenosis: stent retriever versus contact aspiration. J Neurosurg 2019;132:1202-1208.

61. Kang DH, Hwang YH. Frontline contact aspiration treatment for emergent large vessel occlusion: a review focused on practical techniques. J Stroke 2019;21:10-22.

62. Tsang A, Orru E, Klostranec JM, Yang IH, Lau KK, Tsang F, et al. Thrombectomy outcomes of intracranial atherosclerosis-related occlusions. Stroke 2019;50:1460-1466.

63. Kang DH, Kim YW, Hwang YH, Park SP, Kim YS, Baik SK. Instant reocclusion following mechanical thrombectomy of in situ thromboocclusion and the role of low-dose intra-arterial tirofiban. Cerebrovasc Dis 2014;37:350-5.

64. Zhao W, Che R, Shang S, Wu C, Li C, Wu L, et al. Low-dose tirofiban improves functional outcome in acute ischemic stroke patients treated with endovascular thrombectomy. Stroke 2017;48:3289-3294.

65. Kim YW, Sohn SI, Yoo J, Hong JH, Kim CH, Kang DH, et al. Local tirofiban infusion for remnant stenosis in large vessel occlusion: tirofiban ASSIST study. BMC Neurol 2020;20:284.

66. Woo HG, Sunwoo L, Jung C, Kim BJ, Han MK, Bae HJ, et al. Feasibility of permanent stenting with solitaire FR as a rescue treatment for the reperfusion of acute intracranial artery occlusion. AJNR Am J Neuroradiol 2018;39:331-336.

67. Li W, Lin L, Zhang M, Wu Y, Liu C, Li $X$, et al. Safety and preliminary efficacy of early tirofiban treatment after alteplase in acute ischemic stroke patients. Stroke 2016;47:2649-2651.

68. Coller BS. Platelets and thrombolytic therapy. N Engl J Med 1990;322:33-42.

69. Adeoye O, Sucharew H, Khoury J, Vagal A, Schmit PA, Ewing I, et al. Combined approach to lysis utilizing eptifibatide and recombinant tissue-type plasminogen activator in acute ischemic stroke-full dose regimen stroke trial. Stroke 2015;
46:2529-2533.

70. Cervo A, Ferrari F, Barchetti G, Quilici L, Piano M, Boccardi E, et al. Use of cangrelor in cervical and intracranial stenting for the treatment of acute ischemic stroke: a "real life" single-center experience. AJNR Am J Neuroradiol 2020;41:20942099.

71. Elhorany M, Lenck S, Degos V, Sourour NA, Frasca Polara G, Shotar $E_{1}$ et al. Cangrelor and stenting in acute ischemic stroke: monocentric case series. Clin Neuroradiol 2021;31: 439-448.

72. Bang OY. Intracranial atherosclerosis: current understanding and perspectives. J Stroke 2014;16:27-35.

73. Park H, Baek JH, Kim BM. Endovascular treatment of acute stroke due to intracranial atherosclerotic stenosis-related large vessel occlusion. Front Neurol 2019;10:308.

74. Baek JH, Kim BM, Heo JH, Kim DJ, Nam HS, Kim YD, et al. Association between flat-panel computed tomography hyperattenuation and clinical outcome after successful recanalization by endovascular treatment. J Neurosurg 2021;135: 704-711.

75. Mori T, Fukuoka M, Kazita K, Mori K. Follow-up study after intracranial percutaneous transluminal cerebral balloon angioplasty. AJNR Am J Neuroradiol 1998;19:1525-1533.

76. Miao Z, Song L, Liebeskind DS, Liu L, Ma N, Wang Y, et al. Outcomes of tailored angioplasty and/or stenting for symptomatic intracranial atherosclerosis: a prospective cohort study after SAMMPRIS. J Neurointerv Surg 2015;7:331-335.

77. Yaghi $S$, Khatri $P$, de Havenon $A$, Yeatts $S$, Chang AD, Cutting $S$, et al. Peri-procedural stroke or death in stenting of symptomatic severe intracranial stenosis. J Neurointerv Surg 2020; 12:374-379.

78. Yu SC, Leung TW, Lee KT, Wong LK. Angioplasty and stenting of intracranial atherosclerosis with the Wingspan system: 1-year clinical and radiological outcome in a single Asian center. J Neurointerv Surg 2014;6:96-102.

79. Vajda Z, Schmid E, Güthe T, Klötzsch C, Lindner A, Niehaus L, et al. The modified Bose method for the endovascular treatment of intracranial atherosclerotic arterial stenoses using the Enterprise stent. Neurosurgery 2012;70:91-101.

80. Alexander MJ, Zauner A, Chaloupka JC, Baxter B, Callison RC, Gupta $R$, et al. WEAVE trial: final results in 152 on-label patients. Stroke 2019;50:889-894.

81. Stracke CP, Fiehler J, Meyer L, Thomalla G, Krause LU, Lowens $\mathrm{S}$, et al. Emergency intracranial stenting in acute stroke: predictors for poor outcome and for complications. J Am Heart Assoc 2020;9:e012795.

82. Baek JH, Kim BM, Kim DJ, Heo JH, Nam HS, Yoo J. Stenting as a rescue treatment after failure of mechanical thrombec- 
tomy for anterior circulation large artery occlusion. Stroke 2016;47:2360-2363.

83. Chang Y, Kim BM, Bang OY, Baek JH, Heo JH, Nam HS, et al. Rescue stenting for failed mechanical thrombectomy in acute ischemic stroke: a multicenter experience. Stroke 2018;49:958-964.

84. Baracchini $C$, Farina $F$, Soso $M$, Viaro $F$, Favaretto $S$, Palmieri $A$, et al. Stentriever thrombectomy failure: a challenge in stroke management. World Neurosurg 2017;103:57-64.

85. Cornelissen SA, Andersson T, Holmberg A, Brouwer PA, Söderman $M$, Bhogal $P$, et al. Intracranial stenting after failure of thrombectomy with the emboTrap device. Clin Neuroradiol 2019;29:677-683.

86. Peng F, Wan J, Liu W, Huang W, Wang L, Qiu T, et al. Efficacy and safety of rescue stenting following failed mechanical thrombectomy for anterior circulation large vessel occlusion: propensity score analysis. J Neurointerv Surg 2020;12:271-273.

87. Premat $K$, Dechartres A, Lenck $S$, Shotar E, Le Bouc R, Degos $V$, et al. Rescue stenting versus medical care alone in refractory large vessel occlusions: a systematic review and meta-analysis. Neuroradiology 2020;62:629-637.

88. Duan G, Feng Z, Zhang L, Zhang P, Chen L, Hong B, et al. Solitaire stents for the treatment of complex symptomatic intracranial stenosis after antithrombotic failure: safety and efficacy evaluation. J Neurointerv Surg 2016;8:680-684.

89. Lee KY, Chen DY, Hsu HL, Chen CJ, Tseng YC. Undersized angioplasty and stenting of symptomatic intracranial tight stenosis with Enterprise: evaluation of clinical and vascular outcome. Interv Neuroradio/ 2016;22:187-195.

90. Huang CM, Hong YF, Xing SH, Xu K, Xu CK, Zhang WJ, et al. Thirty-day outcomes of the enterprise stent in treating hy- poperfusion of symptomatic intracranial stenosis. World Neurosurg 2019;129:e429-e435.

91. Salik AE, Selcuk HH, Zalov H, Kilinc F, Cirak M, Kara B. Medium-term results of undersized angioplasty and stenting for symptomatic high-grade intracranial atherosclerotic stenosis with Enterprise. Interv Neuroradiol 2019;25:484-490.

92. Feng $Z$, Duan $G$, Zhang $P$, Chen $L, X u Y$, Hong $B$, et al. Enterprise stent for the treatment of symptomatic intracranial atherosclerotic stenosis: an initial experience of 44 patients. BMC Neurol 2015;15:187.

93. Vajda Z, Güthe T, Perez MA, Kurre W, Schmid E, Bäzner $H_{\text {, et }}$ al. Prevention of intracranial in-stent restenoses: predilatation with a drug eluting balloon, followed by the deployment of a self-expanding stent. Cardiovasc Intervent Radiol 2013;36:346-352.

94. Xu H, Quan T, Zaidat O0, Chen D, Wang Z, Yuan Y, et al. Neuroform EZ stenting for symptomatic intracranial artery stenosis: 30 days outcomes in a high-volume stroke center. Front Neurol 2019;10:428.

95. Sweid A, Herial N, Sajja K, Chalouhi N, Velagapudi L, Doermann $A$, et al. Early multicenter experience with the Neuroform Atlas stent: feasibility, safety, and efficacy. Neurosurgery 2020;87:E321-E335.

96. Miao Z, Zhang Y, Shuai J, Jiang C, Zhu , $_{\text {, Chen }} \mathrm{K}_{1}$ et al. Thirty-day outcome of a multicenter registry study of stenting for symptomatic intracranial artery stenosis in China. Stroke 2015;46:2822-2829.

97. Hassan $A E$, Mohammaden $M H$, Rabah RR, Tekle WG. Initial experience with the next-generation resolute onyx zotarolimus-eluting stent in symptomatic intracranial atherosclerotic disease. Front Neurol 2020;11:570100. 